\title{
Editorial: From Whole-Cell to Single Synapse Engrams - Breaking the Code for Memory Formation, Storage and Recall
}

\author{
Antonino Cattaneo ${ }^{1 *}$ and Marco Mainardi ${ }^{1,2 *}$ \\ ${ }^{1}$ Laboratory of Biology "Bio@SNS”, Scuola Normale Superiore, Pisa, Italy, ${ }^{2}$ Neuroscience Institute, National Research \\ Council, Pisa, Italy
}

Keywords: memory engrams, synaptic plasticity, learning, dendritic spines, long-term potentiation

Editorial on the Research Topic

From Whole-Cell to Single Synapse Engrams - Breaking the Code for Memory Formation, Storage and Recall

The capability of animal brains to process, store, and recall specific information is essential to express acquired survival habits, such as repeating the same path to reach a food-rich area, or to keep out of predators. In Man, the emergence of language and culture have pushed these functions up to defining our individual and collective identities. The rooting of our self in memory is, of course, epitomized by Alzheimer's disease, in which impaired memory formation and recall deprive

OPEN ACCESS

Edited and reviewed by: Jason D. Shepherd,

The University of Utah, United States

${ }^{*}$ Correspondence:

Antonino Cattaneo antonino.cattaneo@sns.it Marco Mainardi marco.mainardi@in.cnr.it

Specialty section:

This article was submitted to Neuroplasticity and Development, a section of the journal

Frontiers in Molecular Neuroscience

Received: 29 December 2021

Accepted: 04 February 2022

Published: 01 March 2022

Citation:

Cattaneo A and Mainardi M (2022)

Editorial: From Whole-Cell to Single

Synapse Engrams - Breaking the Code for Memory Formation, Storage

and Recall.

Front. Mol. Neurosci. 15:845516.

doi: 10.3389/fnmol.2022.845516 an individual of its ties, progressively cutting all the bridges with past experience.

Understanding the phenomenology and mechanisms of information processing has been a central problem from the very foundations of modern neuroscience (Sherrington, 1906; Adrian, 1947; Hebb, 1949). Currently, the study of memory gravitates around engrams, i.e., the cellular substrates necessary and sufficient for memory storage and recall (Josselyn and Tonegawa, 2020). Notably, the multiplicity of conceptual and operative definitions about the different modalities, phases and processes associated with memory can be confusing: the Perspective by Hardt and Sossin puts some much needed order on this matter.

Memory engrams are currently conceived as cell assemblies, with their whole-neuron spatial scale being dictated also by limiting technical and experimental reasons. However, decades of studies have shown that the neuronal soma collects inputs from a multitude of synapses subject to individual (and rather independent) structural and functional plasticity processes. Synaptic plasticity is recognized to underlie learning and memory and, according to the synaptic plasticity and memory hypothesis, it is necessary and sufficient for the storage of information required for the type of memory mediated by the brain area in which that plasticity is observed (Takeuchi et al., 2014).

The gap between the whole-neuron and the synaptic scales opens the question about the existence of synaptic engrams, prompting the implementation of appropriate methods for the visualization, analysis and manipulation of individual synapses undergoing learning-related plasticity. Such methods are indeed starting to appear (Hayashi-Takagi et al., 2015; Gobbo et al., 2017), and are the subject of the Review by Gobbo and Cattaneo.

The possibility to affect an acquired behavior by specifically acting on the synapses potentiated during the learning phase of a task (Hayashi-Takagi et al., 2015) demonstrates that analyzing engrams at the whole-cell scale could be missing the finer details of the picture. The Original Research Article by Perez-Alvarez et al. presents experiments on organotypic brain slices using 
a custom-made microscope and a tubulin-bound version of the CaMPARI2 $\mathrm{Ca}^{2+}$ sensor, TubuTag, showing the existence of a dendritic shaft $\mathrm{Ca}^{2+}$ gradient in response to focal stimulation. Even though TubuTag cannot visualize individual dendritic spines, the results suggest a possible mechanism for the generation of clusters of potentiated dendritic spines, which have been also observed, for instance, in fixed mouse hippocampi (Gobbo et al., 2017).

Of course, clustering of potentiated synapses is not a trivial byproduct of $\mathrm{Ca}^{2+}$ (or potentiation-related protein) spillover out of the stimulated site, but represents an efficient way to implement the associativity and cooperativity rules of synaptic plasticity, as well as to integrate multiple synaptic inputs. The Review by Kastellakis and Poirazi describes the different faces of synapse clustering, how it can be achieved, and its relevance for the pathophysiology of brain circuits, with a careful analysis of its cellular-molecular basis, along with computational modeling studies aimed at extracting the rules for and the advantages of grouping potentiated synapses together.

Variations and rearrangements in the protein content of synapses provide the molecular basis for synaptic potentiation; at glutamatergic synapses, which mediate most of the brain's excitatory neurotransmission, the addition of new neurotransmitter receptor units to obtain an increase of synaptic currents (Diering and Huganir, 2018) is an evident example of molecular plasticity. However, there is much more to synaptic potentiation. By concentrating on PSD-95, researchers showed a remarkable diversity in its interactome, with large supramolecular complexes comprising cytoskeletal elements, adaptors, kinases and phosphatases (Fernandez et al., 2009). On the other hand, a full understanding of the relationship between protein composition and synapse functional state(s) is complicated by the heterogeneity of the synaptic repertoire of the brain, which is not taken into account by the vast majority of approaches for studying synapse composition (Grant and Fransen, 2020). A step toward this direction is the combination of regulatory sequences from the mRNA of the immediate early-gene Arc (as used by Hayashi-Takagi et al., 2015) with an oligopeptide enhancing synaptic localization to achieve in vivo, activity-dependent local translation at potentiated dendritic spines of genetically encoded reporters or actuators, such as channelrhodopsins or proteomic probes (Gobbo et al., 2017; Mainardi et al., 2019).

\section{REFERENCES}

Adrian, E. D. (1947). The Physical Background of Perception. Oxford: Clarendon Press.

Diering, G. H., and Huganir, R. L. (2018). The AMPA receptor code of synaptic plasticity. Neuron 100, 314-329. doi: 10.1016/j.neuron.2018.10.018

Fernandez, E., Collins, M. O., Uren, R. T., Kopanitsa, M. V., Komiyama, N. H., Croning, M. D., et al. (2009). Targeted tandem affinity purification of PSD-95 recovers core postsynaptic complexes and schizophrenia susceptibility proteins. Mol Syst Biol 5, 269. doi: 10.1038/msb.2009.27

Gobbo, F., Marchetti, L., Jacob, A., Pinto, B., Binini, N., Pecoraro Bisogni, F., et al. (2017). Activity-dependent expression of Channelrhodopsin at neuronal synapses. Nat. Commun. 8, 1629. doi: 10.1038/s41467-017-01699-7
These state-dependent proteomic studies will ultimately provide the molecular fingerprint associated to information storage at potentiated synapses. The Hypothesis article by Goult speculates that a molecular code for memory storage exists, and describes a provocative possibility of a binary code centered around scaffolding proteins. Abundant experimental effort will be required to prove whether this speculative code actually exists.

An additional aspect in the elucidation of the neural basis for engram formation is the contribution of inhibitory (inter)neurons to memory-related synaptic circuits and cellular ensembles. Interestingly, inhibitory neurons express a repertoire of immediate-early genes closely matching excitatory neurons, including cFos. Moreover, different classes of inhibitory neurons appear to have specific roles in different phases of memory. Finally, GABAergic circuits are thought to participate in keeping memory traces latent. These important issues are discussed in the Review by Giorgi and Marinelli.

We are confident that the different contributions of this Research Topic provide an overview and a primer on the most relevant issues and unaddressed questions in memory engram research. We also believe that experimental efforts should concentrate on (i) defining the molecular identity of synapses in a state-specific manner (i.e., basal activity, short- and longterm potentiation); (ii) mapping the distribution of synapses potentiated during the different phases of a learning and memory recall task to provide reliable datasets for the elaboration of computational models; (iii) addressing the relationship between cellular memory engrams and bona fide synaptic engrams. All these points are relevant to basic neurophysiology, but might also provide a strong knowledge basis for a substantial advancement toward the elaboration of therapeutic approaches to developmental and neurodegenerative synaptopathies.

\section{AUTHOR CONTRIBUTIONS}

$\mathrm{AC}$ and $\mathrm{MM}$ wrote, revised the manuscript, and approved it for publication.

\section{FUNDING}

This work was supported by the Grant PRIN 2017 2017HPTFFC from the Italian Ministry of University and Research to AC.

Grant, S. G. N., and Fransen, E. (2020). The synapse diversity dilemma: molecular heterogeneity confounds studies of synapse function. Front. Synaptic Neurosci. 12, 590403. doi: 10.3389/fnsyn.2020.590403

Hayashi-Takagi, A., Yagishita, S., Nakamura, M., Shirai, F., Wu, Y. I., Loshbaugh, A. L., et al. (2015). Labelling and optical erasure of synaptic memory traces in the motor cortex. Nature 525, 333-338. doi: 10.1038/nature15257

Hebb, D. O. (1949). The Organization of Behavior; A Neuropshychological Theory. New York: Wiley.

Josselyn, S. A., and Tonegawa, S. (2020). Memory engrams: recalling the past and imagining the future. Science 2020, 367. doi: 10.1126/science.aaw 4325

Mainardi, M., Gobbo, F., Jacob, A., Faraone, A., Sorokina, O., Zentilin, L., et al. (2019). "Proteomic characterization of the PSD-95 interactome selectively at in 
vivo potentiated synapses," in ProMEMO - Proteins and Circuits in Memory. (Copenaghen, Denmark, 5-7 March).

Sherrington, C. S. (1906). The Integrative Action of the Nervous System. New York: C Scribner and Sons.

Takeuchi, T., Duszkiewicz, A. J., and Morris, R. G. (2014). The synaptic plasticity and memory hypothesis: encoding, storage and persistence. Philos. Trans. R. Soc. Lond. B. Biol. Sci. 369, 20130288. doi: 10.1098/rstb.2013. 0288

Conflict of Interest: The authors declare that the research was conducted in the absence of any commercial or financial relationships that could be construed as a potential conflict of interest.
Publisher's Note: All claims expressed in this article are solely those of the authors and do not necessarily represent those of their affiliated organizations, or those of the publisher, the editors and the reviewers. Any product that may be evaluated in this article, or claim that may be made by its manufacturer, is not guaranteed or endorsed by the publisher.

Copyright $\odot 2022$ Cattaneo and Mainardi. This is an open-access article distributed under the terms of the Creative Commons Attribution License (CC BY). The use, distribution or reproduction in other forums is permitted, provided the original author(s) and the copyright owner(s) are credited and that the original publication in this journal is cited, in accordance with accepted academic practice. No use, distribution or reproduction is permitted which does not comply with these terms. 